11 Klok FA, Kruisman E, Spaan J, et al. Comparison of the revised Geneva score with the Wells rule for assessing clinical probability of pulmonary embolism. J Thromb Haemost 2008; 6: 40-44.

12 van Belle A, Buller HR, Huisman MV, et al. Effectiveness of managing suspected pulmonary embolism using an algorithm combining clinical probability, D-dimer testing, and computed tomography. JAMA 2006; 295: 172-179.

\title{
Assessment of ventriculo-arterial interaction in pulmonary arterial hypertension using wave intensity analysis
}

To the Editor:

Wave intensity analysis (WIA) is a recently described haemodynamic analysis methodology which enables assessment of ventriculo-arterial interactions via time-domain analysis of pressure and flow waveforms [1]. It enables quantification of wave energy, separation of waves into forward and backward components, and estimation of wave speed [2]. To date, WIA has not been applied to the pulmonary arterial circulation in humans. As WIA offers a potential novel approach for the study of pulmonary haemodynamics, the present study sought to evaluate the feasibility of invasive WIA of the pulmonary circulation during right heart catheterisation (RHC).

The study was approved by the institutional review board (Sydney Local Health District Ethics Review Committee (RPA Zone)) and consent was obtained from seven controls (mean \pm SD age $69 \pm 9$ years, three females) and six patients (age 56 \pm 13 years, four females) with pulmonary arterial hypertension (PAH). Control subjects presented to the cardiac catheterisation laboratory for investigation of possible coronary artery disease. Following standard pulmonary haemodynamic measurements (including thermodilution cardiac output), a combined dual-tipped pressure and Doppler wire (ComboWire; Volcano, Rancho Cordova, CA, USA) was positioned distal to the origin of the right or left lower lobe pulmonary artery via a 6 Fr multipurpose catheter (Mach 1; Boston Scientific, Natick, MA, USA) under angiographic guidance. Pressure and flow measurements were acquired simultaneously at a sampling frequency of $1000 \mathrm{~Hz}$, once stable signals were obtained.

Data were processed offline using customised MatLab software (MathWorks, Natick, MA, USA). Signals were ensemble-averaged with timing gated to the ECG R-wave and smoothed using a Savitzky-Golay filter [3]. Intrinsic hardware processing delays (between pressure and flow signals) were corrected by shifting signals to achieve a linear pressure-flow (P-U) relationship in early systole [4]. Wave speed (c) was derived from the P-U loop method [2]:

$$
\mathrm{c}=(\mathrm{dP} / \mathrm{dU}) / \rho
$$

where $\mathrm{dP} / \mathrm{dU}$ is the slope of $\mathrm{P}-\mathrm{U}$ loop in early systole and $\rho$ is density of blood, assumed to be $1060 \mathrm{~kg} \cdot \mathrm{m}^{-3}$.

WIA was performed as previously described [3] with separation of waves into forward $\left(\mathrm{dI}_{+}\right)$and backward (dI_) wave intensities:

$$
\begin{aligned}
& \mathrm{dI}_{+}=+(\mathrm{dP} / \mathrm{dt}+\rho \mathrm{c} \times \mathrm{dU} / \mathrm{dt})^{2} / 4 \rho c \\
& \mathrm{dI}_{-}=-(\mathrm{dP} / \mathrm{dt}-\rho \mathrm{c} \times \mathrm{dU} / \mathrm{dt})^{2} / 4 \rho c
\end{aligned}
$$

Total wave energy for separated waves was calculated by integration of wave intensities over time. The magnitude of wave reflection (reflection coefficient) was calculated as the ratio of the total forward compression wave intensity to the total backward compression wave intensity [5]. The reflection distance (L) from the measurement site was estimated from the time delay between the peaks of the forward and backward compression waves, together with knowledge of wave speed:

$$
\mathrm{L}=\mathrm{c} \Delta \mathrm{t} / 2
$$

Results are presented as mean $\pm \mathrm{SD}$, unless otherwise stated. Differences between groups were compared using the Mann-Whitney U-test. Correlation was performed using Pearson's test. Significance was inferred at a two-sided $\mathrm{p}<0.05$. 

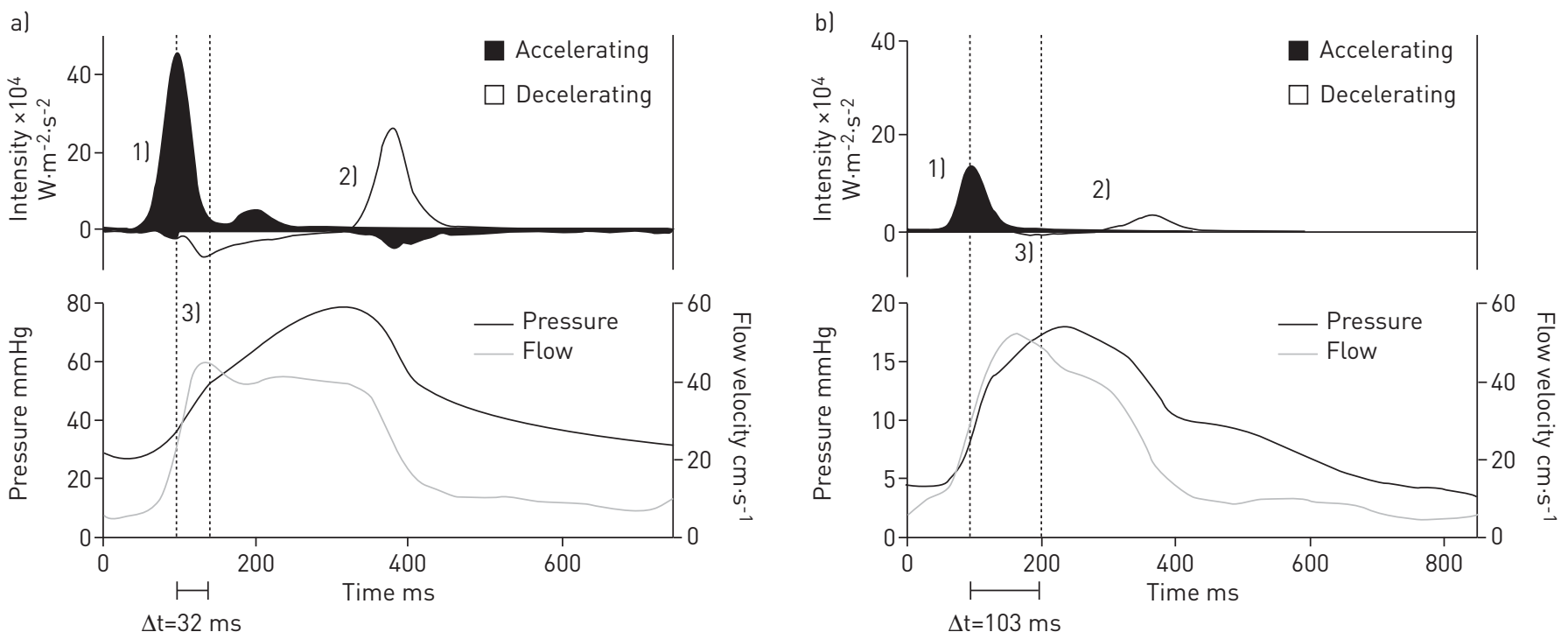

FIGURE 1 Wave intensity analysis and corresponding ensemble-averaged pressure and flow velocity waveforms over a cardiac cycle. Positive wave intensity indicates forward travelling waves and negative intensity indicates backward travelling waves. The three dominant waves that were consistently identified included: 1) a forward compression wave (FCW), 2) forward expansion wave, and 3) backward compression wave (BCW). Black waves accelerate forward flow and white waves decelerate forward flow. $\Delta \mathrm{t}$ refers to the time delay between incident FCW and reflected BCW. a) Pulmonary arterial hypertension (PAH) subject with mean pulmonary arterial pressure (PAP) of $40 \mathrm{mmHg}$ and pulmonary vascular resistance (PVR) of 6 Wood units. Wave intensity analysis revealed significant wave reflection (reflection coefficient 0.26 ) with BCW arriving during the FCW in mid-systole. Estimated wave speed was $5.8 \mathrm{~m} \cdot \mathrm{s}^{-1}$. $\mathrm{b}$ ) Control subject with mean PAP of $14 \mathrm{mmHg}$ and PVR of 0.9 Wood units. In contrast to the PAH subject, wave reflection was minimal (reflection coefficient 0.08 ) with the BCW arriving after the FCW. The estimated wave speed was $3.5 \mathrm{~m} \cdot \mathrm{s}^{-1}$.

The aetiologies of PAH were idiopathic PAH $(n=3)$, scleroderma PAH $(n=2)$ and portopulmonary hypertension $(n=1)$. Haemodynamics in PAH group revealed a mean pulmonary arterial pressure (PAP) of $41 \pm 5 \mathrm{mmHg}$, pulmonary vascular resistance (PVR) of $6.3 \pm 4.4$ Wood units and pulmonary capillary wedge pressure (PCWP) of $8 \pm 3 \mathrm{mmHg}$. Controls had normal pulmonary haemodynamics (mean PAP of $16 \pm 5 \mathrm{mmHg}$, PVR of $1.3 \pm 0.7 \mathrm{mmHg}$, and PCWP of $8 \pm 3 \mathrm{mmHg}$ ).

In both control and PAH groups, three dominant waves were consistently identified: 1) a forward compression wave (FCW) in early systole corresponding to ventricular ejection; 2) a forward expansion wave (FEW) in late systole representing ventricular relaxation and deceleration of blood flow; and 3) a backward compression wave (BCW) related to downstream reflection of the incident FCW (fig. 1).

PAH patients displayed an increase in the total intensity in all of the identified waves (total FCW of $164.5 \pm 39.7 \times 10^{2} \mathrm{~W} \cdot \mathrm{m}^{-2} \cdot \mathrm{s}^{-1}$ versus $88.3 \pm 20.7 \times 10^{2} \mathrm{~W} \cdot \mathrm{m}^{-2} \cdot \mathrm{s}^{-1}$ in controls; total FEW of $85.1 \pm 36.0 \times 10^{2} \mathrm{~W} \cdot \mathrm{m}^{-2} \cdot \mathrm{s}^{-1}$ versus $32.5 \pm 13.7 \times 10^{2} \mathrm{~W} \cdot \mathrm{m}^{-2} \cdot \mathrm{s}^{-1}$ in controls; all $\mathrm{p}<0.01$ ), with the most notable difference being a five-fold increase in the total BCW wave intensity (total BCW of $56.9 \pm 14.6 \mathrm{~W} \cdot \mathrm{m}^{-2} \cdot \mathrm{s}^{-1}$ versus $10.7 \pm 5.6 \mathrm{~W} \cdot \mathrm{m}^{-2} \cdot \mathrm{s}^{-1}$ in controls; $\left.\mathrm{p}<0.01\right)$. Importantly, the total $\mathrm{BCW}$ intensity was proportionally larger relative to the total FCW intensity in PAH patients, resulting in a higher reflection coefficient $(0.35 \pm 0.05$ versus $0.11 \pm 0.06 ; \mathrm{p}<0.01)$. Wave speed was significantly faster in PAH patients $\left(6.9 \pm 1.3 \mathrm{~m} \cdot \mathrm{s}^{-1}\right.$ versus $\left.3.8 \pm 1.0 \mathrm{~m} \cdot \mathrm{s}^{-1} ; \mathrm{p}<0.01\right)$, leading to earlier arrival $(45 \pm 20 \mathrm{~ms}$ versus $89 \pm 30 \mathrm{~ms} ; \mathrm{p}<0.01)$ of the reflected $\mathrm{BCW}$ in midsystole. The estimated reflection site in PAH patients was $15 \pm 5 \mathrm{~cm}$ downstream from the measurement site in the proximal lower lobe artery. Wave speed correlated significantly with pulmonary capacitance $(\mathrm{r}=0.7, \mathrm{p}<0.01)$.

To the best of our knowledge, this is the first study to apply WIA for the evaluation of pulsatile wave travel in the human pulmonary arterial circulation. WIA was feasible during routine RHC using a commercially available dual-tippled pressure and Doppler wire, and revealed relevant pathophysiological changes in PAH subjects characterised by: 1) increased wave reflections due to distal closed-end reflection sites; 2) increased energies of travelling waves indicating increased right ventricular workload; and 3) higher wave speed due to arterial stiffening resulting in earlier arrival of large reflected waves, augmenting afterload unfavourably in the still-ejecting right ventricle. The potential appeal of WIA is that these pathophysiological changes can be quantified in an integrative time-domain approach.

Time-domain analysis of pulmonary artery waveforms using invasive high-fidelity measurements has previously been limited to pressure waveforms only [6]. It has been suggested that pulmonary artery 
pressure waveform analysis can assess the timing, extent and magnitude of wave reflections [7]. However, time-domain pressure waveform analysis is critically dependent on the accurate determination of the inflection point, which may not be identifiable in all patients [8] and may, thus, result in erroneous estimation of wave reflection indices [9]. By combining pressure and flow waveforms, WIA overcomes such limitations of isolated pulmonary artery pressure waveform analysis, and potentially provides an improved assessment of wave reflection. Furthermore, WIA enables determination of local wave speed, allowing estimation of reflection site distances. However, it is unknown, at present, whether WIA can discriminate differences in reflection sites in chronic thromboembolic pulmonary hypertension (CTEPH) versus PAH, and whether it can aid in the partitioning of predominantly proximal versus distal disease in CTEPH.

Pulmonary artery impedance analysis has been the traditional method used to fully characterise pulsatile right ventricular afterload $[10,11]$. As the impedance spectrum is presented in the frequency-domain, interpretation is not straightforward and is unintuitive for most clinicians. WIA offers an alternate approach to impedance analysis, and it has been demonstrated that both impedance and WIA can provide similar results for the separation of forward and backward waveforms [12], with WIA having the distinct advantage of data presentation in the time-domain. However, impedance and WIA are not interchangeable in other aspects. WIA describes wave energy which is a function of both the pump and the vasculature, whereas impedance provides information regarding right ventricular afterload (or the arterial system).

Some methodological limitations need to be acknowledged. Obtaining high quality in vivo measurements was challenging and it was technically difficult to achieve a stable catheter position in the main pulmonary artery (the ideal anatomical position as the entire circulation is "seen"); therefore, the lower lobe vessels were studied. It is unknown whether WIA (or impedance) will differ between the upper or lower lobe pulmonary artery in healthy and PAH subjects in the supine posture, as we are not aware of any previous literature on this subject. Numbers of subjects were small in this preliminary study, but the differences between groups were very marked.

In conclusion, we demonstrate, in this preliminary "proof of principle" study, that WIA of the pulmonary circulation is feasible during RHC and may offer a novel time-domain based approach for the assessment of ventriculo-arterial interactions in pulmonary vascular disease. Further validation studies are required to determine whether parameters derived from WIA provide incremental clinical information, additional to conventional haemodynamics, regarding prognosis and response to therapy.

0 @ERSpublications

Demonstration that wave intensity analysis of the pulmonary circulation is feasible during right heart catheterisation http://ow.ly/rH5bE

Edmund M.T. Lau ${ }^{1,2,3}$, David Abelson ${ }^{2}$, Nathan Dwyer ${ }^{4}$, Young Yu ${ }^{1,2}$, Martin K. Ng ${ }^{1,2}$ and David S. Celermajer ${ }^{1,2}$

${ }^{1}$ Sydney Medical School, University of Sydney, Camperdown, ${ }^{2}$ Dept of Cardiology, Royal Prince Alfred Hospital, Camperdown, ${ }^{3}$ Dept of Respiratory Medicine, Royal Prince Alfred Hospital, Camperdown, and ${ }^{4}$ Dept of Cardiology, Royal Hobart Hospital, Hobart, Australia.

Correspondence: D.S. Celermajer, Dept of Cardiology, Royal Prince Alfred Hospital, Missenden Road, Camperdown, NSW 2050, Australia. E-mail: david.celermajer@email.cs.nsw.gov.au

Received: Aug 252013 | Accepted after revision: Dec 032013 | First published online: Jan 162014

Support statement: National Health and Medical Research Council Project Grant 512404 and Royal Australasian College of Physicians Basser Scholarship.

Conflict of interest: None declared.

\section{References}

Parker KH, Jones CJ. Forward and backward running waves in the arteries: analysis using the method of characteristics. J Biomech Eng 1990; 112: 322-326.

Parker KH. An introduction to wave intensity analysis. Med Biol Eng Comput 2009; 47: 175-188.

Davies JE, Whinnett ZI, Francis DP, et al. Evidence of a dominant backward-propagating "suction" wave responsible for diastolic coronary filling in humans, attenuated in left ventricular hypertrophy. Circulation 2006; 113: $1768-1778$.

4 Dwyer N, Yong AC, Kilpatrick D. Variable open-end wave reflection in the pulmonary arteries of anesthetized sheep. J Physiol Sci 2012; 62: 21-28.

5 Penny DJ, Mynard JP, Smolich JJ. Aortic wave intensity analysis of ventricular-vascular interaction during incremental dobutamine infusion in adult sheep. Am J Physiol Heart Circ Physiol 2008; 294: H481-H489.

6 Champion HC, Michelakis ED, Hassoun PM. Comprehensive invasive and noninvasive approach to the right ventricle-pulmonary circulation unit: state of the art and clinical and research implications. Circulation 2009; 120 : 992-1007. 
7 Nakayama Y, Nakanishi N, Hayashi T, et al. Pulmonary artery reflection for differentially diagnosing primary pulmonary hypertension and chronic pulmonary thromboembolism. J Am Coll Cardiol 2001; 38: 214-218.

8 Castelain V, Hervé P, Lecarpentier Y, et al. Pulmonary artery pulse pressure and wave reflection in chronic pulmonary thromboembolism and primary pulmonary hypertension. J Am Coll Cardiol 2001; 37: 1085-1092.

9 Hughes AD, Park C, Davies J, et al. Limitations of augmentation index in the assessment of wave reflection in normotensive healthy individuals. PLoS One 2013; 8: e59371.

10 Huez S, Brimioulle S, Naeije R, et al. Feasibility of routine pulmonary arterial impedance measurements in pulmonary hypertension. Chest 2004; 125: 2121-2128.

11 Murgo JP, Westerhof N. Input impedance of the pulmonary arterial system in normal man. Effects of respiration and comparison to systemic impedance. Circ Res 1984; 54: 666-673.

12 Hughes AD, Parker KH. Forward and backward waves in the arterial system: impedance or wave intensity analysis? Med Biol Eng Comput 2009; 47: 207-210.

\title{
Neutrophil gelatinase-associated lipocalin in idiopathic pulmonary fibrosis
}

\begin{abstract}
To the Editor:
Neutrophil gelatinase-associated lipocalin (NGAL) is a $25 \mathrm{kD}$ lipocalin that is covalently bound to matrix metalloproteinase (MMP)-9 produced by neutrophils [1]. NGAL in blood or bronchoalveolar lavage fluid (BALF) may reflect neutrophilic inflammation in the lungs [2, 3], and it is highly induced in injured epithelial cells, including those in the lung [4]. Possible roles for neutrophilic inflammation and epithelial injury have been reported in idiopathic pulmonary fibrosis (IPF). Thus, we hypothesised that NGAL may be associated with the pathogenesis of IPF. To investigate the roles of NGAL in IPF, we used immunohistochemical staining for lung specimens and measured plasma and BALF NGAL levels. Our study was approved by the Ethics Committee of Kyoto University (approval No. E438), and written informed consent was obtained from all study participants.
\end{abstract}

First, we immunohistochemically stained the lung tissue specimens of six IPF patients, two nonspecific interstitial pneumonia (NSIP) patients, and a control (normal area distant from the lesion of surgically diagnosed organising pneumonia) for NGAL using a conventional method [5]. We also performed sequential immunofluorescent staining for NGAL and MMP-9. Immunohistochemical staining showed that NGAL was abundantly expressed in airway epithelial cells that covered the honeycomb cysts in IPF (fig. 1a and b). Further, histologically normal bronchioles in the fibrotic lesions also exhibited abundant NGAL expression, although apparently normal alveolar walls exhibited little NGAL expression. NGAL was also expressed in macrophages, neutrophils and some alveolar epithelial cells.

In NSIP, NGAL was expressed in macrophages and a small number of alveolar epithelial cells. Airway epithelial cells also showed NGAL expression; however, there were fewer positive cells and their staining intensity was weaker compared with that of the IPF specimens (fig. 1c). The control lung specimen revealed an expression pattern similar to that of NSIP patients (fig. 1d). Sequential immunofluorescent staining indicated that NGAL and MMP-9 were similarly expressed in a patchy pattern along the airway epithelial cells, which covered honeycomb cysts in the lung tissues of IPF (fig. 1e and f).

We also measured plasma and BALF NGAL levels for 36 IPF patients (25 males, 27 smokers, 15 with histological diagnosis) who visited Kyoto University Hospital from October 2007 to April 2012 and underwent bronchoalveolar lavage (BAL). IPF was diagnosed on the basis of the official joint statement on IPF published in 2011 [6]. For disease controls, we also enrolled 26 patients as "non-IPF" interstitial pneumonia group (11 males, 14 smokers). The non-IPF group consists of seven patients who had undergone surgical lung biopsy (SLB) (two had NSIP and five exhibited a pathological pattern of bronchiolocentric interstitial pneumonia) and 19 patients with interstitial pneumonia of unknown cause who did not undergo SLB but exhibited "inconsistent with UIP pattern" on high-resolution computed tomography [6]. At the time of enrolment, none of these patients had been treated for interstitial lung disease.

Blood and BALF sampling and pulmonary function testing were performed at the same time within 7 days of admission. In addition, 31 subjects without lung disease were enrolled as healthy controls for plasma NGAL determination that were matched for age and serum creatinine levels. Plasma NGAL levels and BALF NGAL levels were determined using sandwich ELIZA (Antibody Shop, Gentofte, Denmark). BALF albumin concentrations were measured by turbidimetric immunoassay (Superior-Microalbumin kit; Mitsubishi 